\title{
C-reactive protein level predicts mortality in COPD: a systematic review and meta-analysis
}

\author{
Giovanni Leuzzi ${ }^{1}$, Carlotta Galeone ${ }^{2}$, Francesca Taverna ${ }^{3}$, Paola Suatoni ${ }^{1}$, \\ Daniele Morelli ${ }^{4}$ and Ugo Pastorino ${ }^{1}$
}

Affiliations: ${ }^{1}$ Thoracic Surgery Unit, IRCCS Istituto Nazionale dei Tumori Foundation, Milan, Italy. ${ }^{2}$ Dept of Clinical Sciences and Community Health, University of Milan, Milan, Italy. ${ }^{3}$ Immunohematology and Transfusion Medicine Service, IRCCS Istituto Nazionale dei Tumori Foundation, Milan, Italy. ${ }^{4}$ Dept of Pathology and Laboratory Medicine, IRCCS Istituto Nazionale dei Tumori Foundation, Milan, Italy.

Correspondence: Giovanni Leuzzi, Thoracic Surgery Unit, IRCCS Istituto Nazionale dei Tumori Foundation, Via Venezian 1, 20133 Milan, Italy. E-mail: giovanni.leuzzi@istitutotumori.mi.it

@ERSpublications

Baseline high CRP level is significantly associated with higher mortality in COPD patients http://ow.ly/iWKb305aYvL

Cite this article as: Leuzzi G, Galeone C, Taverna F, et al. C-reactive protein level predicts mortality in COPD: a systematic review and meta-analysis. Eur Respir Rev 2017; 26: 160070 [https://doi.org/10.1183/ 16000617.0070-2016].

ABSTRACT The prognostic role of baseline C-reactive protein (CRP) in chronic obstructive pulmonary disease (COPD) is controversial. In order to clarify this issue, we performed a systematic review and metaanalysis to assess the predictive effect of baseline CRP level in COPD patients. 15 eligible articles focusing on late mortality in COPD were included in our study. We performed a random-effects meta-analysis, and assessed heterogeneity and publication bias. We pooled hazard ratio (HR) estimates and their 95\% confidence intervals on mortality for the comparison between the study-specific highest category of CRP level versus the lowest category. In overall analysis, elevated baseline CRP levels were significantly associated with higher mortality (HR 1.53, 95\% CI 1.32-1.77, $I^{2}=68.7 \%, \mathrm{p}<0.001$ ). Similar results were observed across subgroups. However, higher mortality risk was reported in studies using a cut-off value of $3 \mathrm{mg} \cdot \mathrm{L}^{-1}$ (HR 1.61, 95\% CI 1.12-2.30) and in those enrolling an Asiatic population (HR 3.51, 95\% CI 1.69-7.31). Our analysis indicates that baseline high CRP level is significantly associated with higher late mortality in patients with COPD. Further prospective controlled studies are needed to confirm these data.

\section{Introduction}

The prevalence of chronic obstructive pulmonary disease (COPD) is $\sim 10 \%$ in adults older than 40 years [1]. According to World Health Organization (WHO) estimates, 65 million people have moderate to severe COPD and more than 3 million people died of COPD in 2005, corresponding to $5 \%$ of all deaths globally [2]. This number is projected to rise by $30 \%$ during the next 10 years and estimates show that COPD will become the third leading cause of death worldwide in 2030 [2]. Approximately $50 \%$ of patients with COPD have at least one exacerbation per year and $>20 \%$ are readmitted within 30 days, with a total of nearly 800000 hospitalisations and USD50 billion in healthcare costs annually [3-5]. COPD is characterised by

This article has supplementary material available from err.ersjournals.com

Received: July 282016 | Accepted after revision: Oct 062016

Conflict of interest: None declared.

Provenance: Submitted article, peer reviewed.

Copyright OERS 2017. ERR articles are open access and distributed under the terms of the Creative Commons Attribution Non-Commercial Licence 4.0. 
persistent airflow limitation that is usually progressive and associated with an enhanced chronic inflammatory response in the airways and the lung to noxious particles or gases [6]. It is well established that COPD is a multifactorial disease composed of both modifiable (smoking, occupational exposures, pollution, etc.) and nonmodifiable (genetics, ageing, bronchial hyperreactivity, etc.) risk factors, with cigarette smoking being the most significant factor [7]. In particular, smokers with COPD have a higher prevalence of respiratory symptoms and lung function abnormalities, a greater annual rate of decline in forced expiratory volume in $1 \mathrm{~s}(\mathrm{FEV} 1)$, and a greater mortality rate compared with nonsmokers [8].

As a rule, the persistent damage related to risk factors causes mucus accumulation, bronchiolar fibrosis and local inflammation (development of lymphoid follicles and inflammatory cells infiltration) [9]. In addition to lung inflammation, it is well recognised that patients with moderate to severe stable COPD frequently demonstrate persistent low-grade systemic inflammation, with elevated levels of circulating molecules that are part of the inflammatory cascade (C-reactive protein (CRP), interleukin (IL)-6, tumour necrosis factor (TNF)- $\alpha$ and blood leukocytes) [10, 11]. This issue is of particular interest when considering that systemic inflammation is a significant risk factor for morbidity or mortality in the general population, especially related to cardiovascular events [12] or cancer onset [13]. Furthermore, the ECLIPSE (Evaluation of COPD Longitudinally to Identify Predictive Surrogate Endpoints) study has recently defined different COPD subtypes, and reported an interesting association between COPD clinical course and systemic inflammation [14].

The search for an optimal prognostic marker in COPD patients is challenging. As defined by McShane et al. [15] and De Gruttola et al. [16], an ideal biomarker must possess several combined properties: 1) a biological role in pathogenesis of disease, 2) easy to measure accurately, 3) sensitive to change in order to be repeatedly evaluated, 4) modifiable by therapies or interventions, 5) a long half-life and 6) associated with outcome. As recently evidenced [17], CRP could be one of the ideal biomarkers available in common clinical practice.

The aim of this meta-analysis was to evaluate and discuss the association between baseline CRP level and mortality in the setting of COPD.

\section{Methods}

We retrieved studies from the PubMed database published up to May 2016 using a web-based search engine. Search terms or related $\mathrm{MeSH}$ terms included "CRP" or "C-reactive protein" or "C reactive protein" and "mortality" or "survival" and "COPD" or "chronic obstructive pulmonary disease" or "smoker" or "smokers" or "smoke". We also manually searched the reference lists of all articles retrieved. Relevant review articles were also cross-referenced.

Two investigators (G.L. and C.G.) independently identified all eligible articles. The first screen was based on article title and then on related abstracts. Subsequently, the full texts of potentially eligible papers were assessed for final inclusion. In order to avoid disagreement in search results, a third reviewer (U.P.) made the final decision in case of different results.

Inclusion criteria were: 1) study enrolling patients with COPD, 2) reported baseline CRP levels, 3) provided information on survival, 4) reported estimates of association between CRP levels and survival in patients with COPD, and 5) inclusion of the most recent article or the most complete article for publication data repeatedly reported by the same author.

Exclusion criteria were: 1) letters, case reports or editorials, 2) abstracts or unpublished studies, 3) animal research, 4) laboratory studies, 5) non-English language articles, 6) articles that measured CRP levels after treatment or discharge and 7) studies not providing all necessary data reported in the inclusion criteria. Review articles were consulted for discussion purposes only. No studies were excluded a priori because of weakness of design or data quality.

In case of incomplete data, we contacted the corresponding authors to acquire the unpublished results. Alternatively, these data were extracted from summary data or the Kaplan-Meier curve according to TIERNEY et al. [18]. If the necessary data were not provided or could not be acquired according to this method, these studies were finally excluded.

Data collected from these studies included first author, year of publication, country of origin, study design, number of institutions involved, recruitment period, sample size, sex, follow-up time, type of COPD (stable or with acute exacerbations), CRP combination with other variables, CRP study-specific cut-off values, outcome (late mortality and early mortality) and mortality risk estimates (hazard ratio (HR) or relative risk) of CRP for survival with their 95\% confidence intervals. Due to the different and nonstandardised outcomes reported in each study, we empirically a priori defined late and early mortality as all causes of deaths occurring after at least and within 24 months, respectively. 
As a rule, we acquired the mortality risk estimates and the corresponding 95\% confidence intervals from results and tables. If a study reported more than one mortality risk estimate, we used the one adjusted for the larger number of available potential confounding factors. When a study reported the adjusted mortality risk estimates and the corresponding p-values, but not the corresponding adjusted 95\% confidence intervals, we calculated the $95 \%$ confidence intervals for a ratio [19]. For studies that reported mortality risk estimates according to a continuous measure of CRP level, we calculated mortality risk estimates and $95 \%$ confidence intervals for an a priori defined cut-off of $10 \mathrm{mg} \cdot \mathrm{L}^{-1}$ [20]. Different cut-off values (i.e. 5 and $15 \mathrm{mg} \cdot \mathrm{L}^{-1}$ ) were used for sensitivity analyses.

The systematic review and meta-analysis was performed and reported according to the MOOSE (Meta-analysis of Observational Studies in Epidemiology) guidelines [21].

\section{Statistical analysis}

We pooled mortality risk estimates for the comparison between the study-specific highest category of CRP level versus the lowest category using the DerSimonian and Laird random-effects model. We used the Duval and Tweedie "trim and fill" method [22] to calculate the effect of potential data censoring or publication bias on the outcome of the meta-analysis. Heterogeneity among studies was assessed using the Chi-squared test and the $I^{2}$ statistic. Usually, $I^{2}<25 \%$ is indicative of low heterogeneity, $I^{2}=25-75 \%$ is indicative of moderate heterogeneity and $I^{2}>75 \%$ is indicative of high heterogeneity. To identify sources of heterogeneity, subgroup analyses were performed according to geographic area (Asia, Europe, America) and CRP study-specific cut-off values ( $\leqslant 3$ versus $>3 \mathrm{mg} \cdot \mathrm{L}^{-1}$ and $\leqslant 5$ versus $>5 \mathrm{mg} \cdot \mathrm{L}^{-1}$ ). Presence of publication bias was assessed by examination of funnel plot and by applying the tests proposed by Egger and Begg.

All the statistical analyses were performed using STATA version 11 (StataCorp, College Station, TX, USA).

\section{Results}

\section{Study selection and characteristics}

Figure 1 summarises the selection process adopted for the systematic review. Overall, 275 studies were identified. By excluding 26 duplicate publications and 147 studies not focusing on the main topics, 102 articles were fully examined. We found 38 studies focusing on CRP and COPD prognosis. 12 studies were excluded because of the following reasons: duplicated data [23, 24], no data on mortality [25-28], insufficient data to calculate $95 \%$ confidence intervals [29-31] and no data on CRP levels used for statistical analysis [32-34]. Finally, 26 articles on COPD mortality were eligible for the meta-analysis [35-60].

Study characteristics of all 26 articles are reported in table 1. Overall, 17890 patients were collected from five retrospective studies $[35,50,51,54,57]$ and 21 prospective studies $[36-49,52,53,55,56,58-60]$. Two

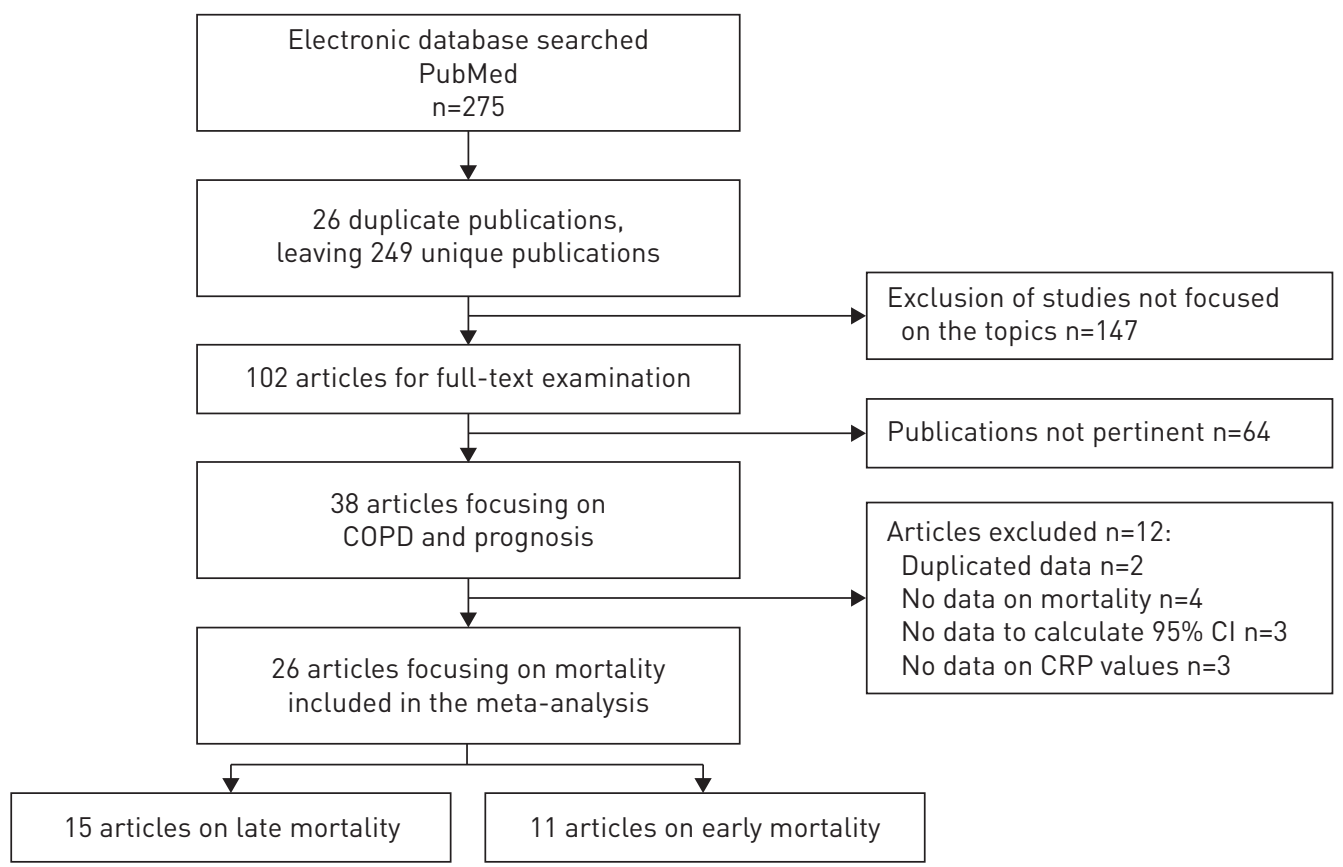

FIGURE 1 Flowchart of literature search and study selection. COPD: chronic obstructive pulmonary disease; CRP: C-reactive protein. 
TABLE 1 Characteristics of studies included in the meta-analysis of association between baseline C-reactive protein (CRP) level and mortality in chronic obstructive pulmonary disease (COPD) patients

\begin{tabular}{|c|c|c|c|c|c|c|c|c|c|}
\hline $\begin{array}{l}\text { First author } \\
\text { [ref.] }\end{array}$ & Year & $\begin{array}{l}\text { Study design; } \\
\text { recruitment period; } \\
\text { location }\end{array}$ & $\begin{array}{c}\text { Patients } \\
\text { n }\end{array}$ & $\begin{array}{c}\text { Type of } \\
\text { patients }\end{array}$ & Main aim & Outcome & CRP & Prognostic value ${ }^{+}$ & $\begin{array}{l}\text { Cut-off } \\
\mathrm{mg} \cdot \mathrm{L}^{-1}\end{array}$ \\
\hline KuLLeR [35] & 1996 & $\begin{array}{l}\text { Retrospective, } \\
\text { multicentric; } \\
\text { 1973-1976; } \\
\text { UK/USA }\end{array}$ & 737 & $\begin{array}{l}\text { Smokers; } \\
\text { stable }\end{array}$ & $\begin{array}{l}\text { To investigate the relationship } \\
\text { between CRP, } \alpha_{1} \text {-acid } \\
\text { glycoprotein and albumin and } \\
\text { subsequent risk of myocardial } \\
\text { infarction and coronary heart } \\
\text { disease death in a nested } \\
\text { case-control study among the } \\
\text { Multiple Risk Factor } \\
\text { Intervention Trial participants }\end{array}$ & $\begin{array}{l}\text { Late } \\
\text { mortality }\end{array}$ & Alone & $\begin{array}{l}\text { Multivariate; } 2.8(1.4-5.4) ; \\
\text { age, tobacco dose, blood } \\
\text { pressure, triglycerides, } \\
\text { cholesterol levels }\end{array}$ & $\begin{array}{l}\text { Not } \\
\text { reported } \\
\text { (fourth } \\
\text { quartile) }\end{array}$ \\
\hline MAN [36] & 2006 & $\begin{array}{l}\text { Prospective, } \\
\text { multicentric; } \\
5 \text { years; } \\
\text { USA/Canada }\end{array}$ & 4803 & $\begin{array}{l}\text { COPD; } \\
\text { stable }\end{array}$ & $\begin{array}{l}\text { To determine whether serum } \\
\text { CRP is associated with } \\
\text { increased risk of all-cause } \\
\text { and disease-specific causes } \\
\text { of mortality, increased risk of } \\
\text { fatal and nonfatal } \\
\text { cardiovascular events, and an } \\
\text { accelerated decline in lung } \\
\text { function in COPD patients }\end{array}$ & $\begin{array}{l}\text { Late } \\
\text { mortality }\end{array}$ & Alone & $\begin{array}{l}\text { Multivariate; } 1.79 \text { (1.25-2.56); } \\
\text { age, sex, race, smoking } \\
\text { status, pack-years, } \\
\text { BMI, FEV1 }\end{array}$ & 7.06 \\
\hline DAHL [37] & 2007 & $\begin{array}{l}\text { Prospective, } \\
\text { multicentric } \\
\text { (epidemiological } \\
\text { study); } \\
\text { 1991-1994; } \\
\text { Denmark }\end{array}$ & 1302 & $\begin{array}{l}\text { COPD; } \\
\text { stable }\end{array}$ & $\begin{array}{l}\text { To determine whether increased } \\
\text { serum CRP in individuals with } \\
\text { airway obstruction predicts } \\
\text { future hospitalisation and } \\
\text { death from COPD }\end{array}$ & $\begin{array}{l}\text { Late } \\
\text { mortality }\end{array}$ & Alone & $\begin{array}{l}\text { Multivariate; } 1.4(1.1-1.8) ; \\
\text { age, sex, tobacco } \\
\text { consumption, FEV1, } \\
\text { cardiovascular disease }\end{array}$ & 3 \\
\hline DE ToRres [38] & 2008 & $\begin{array}{l}\text { Prospective, } \\
\text { multicentric; } \\
\text { 2000-2004; } \\
\text { Spain/USA }\end{array}$ & 218 & $\begin{array}{l}\text { COPD; } \\
\text { stable }\end{array}$ & $\begin{array}{l}\text { To determine if CRP levels are } \\
\text { associated with survival in } \\
\text { patients with moderate to } \\
\text { very severe COPD in } \\
\text { comparison with other } \\
\text { well-known prognostic } \\
\text { parameters }\end{array}$ & $\begin{array}{l}\text { Late } \\
\text { mortality }\end{array}$ & Alone & $\begin{array}{l}\text { Multivariate; } 1.00 \text { (0.82-1.22); } \\
\text { age, sex, pack-years, } \\
\text { cardiovascular disease, } \\
\text { corticosteroids }\end{array}$ & $\begin{array}{l}\text { Not } \\
\text { reported }\end{array}$ \\
\hline Mehrotra [39] & 2010 & $\begin{array}{l}\text { Prospective, } \\
\text { community-based } \\
\text { observational cohort } \\
\text { (Pittsburgh, PA and } \\
\text { Memphis, TN); } \\
\text { 1997-1998; } \\
\text { USA }\end{array}$ & 268 & $\begin{array}{l}\text { COPD; } \\
\text { stable }\end{array}$ & $\begin{array}{l}\text { To identify significant covariates } \\
\text { in addition to spirometry that } \\
\text { predict mortality in elderly } \\
\text { subjects with obstructive } \\
\text { airway disease }\end{array}$ & $\begin{array}{l}\text { Late } \\
\text { mortality }\end{array}$ & Alone & $\begin{array}{l}\text { Multivariate; } 1.12 \text { (0.90-1.30); } \\
\text { age, sex, race, smoking } \\
\text { status, cardiovascular } \\
\text { disease }\end{array}$ & $\begin{array}{l}\text { Not } \\
\text { reported }\end{array}$ \\
\hline LIU [40] & 2011 & $\begin{array}{l}\text { Prospective, } \\
\text { single-institution; } \\
\text { 2005-2006; } \\
\text { China }\end{array}$ & 114 & $\begin{array}{l}\text { COPD; } \\
\text { stable }\end{array}$ & $\begin{array}{l}\text { To investigate the predictive } \\
\text { value of combined serum } \\
\text { CRP and BODE index score } \\
\text { for mortality in COPD patients }\end{array}$ & $\begin{array}{l}\text { Late } \\
\text { mortality }\end{array}$ & Alone & $\begin{array}{l}\text { Multivariate; } 5.15 \text { (1.65-16.60); } \\
\text { BODE index }\end{array}$ & 3 \\
\hline
\end{tabular}




\begin{tabular}{|c|c|c|c|c|c|c|c|c|c|}
\hline $\begin{array}{l}\text { First author } \\
\text { [ref.] }\end{array}$ & Year & $\begin{array}{l}\text { Study design; } \\
\text { recruitment period; } \\
\text { location }\end{array}$ & $\begin{array}{c}\text { Patients } \\
\mathrm{n}\end{array}$ & $\begin{array}{c}\text { Type of } \\
\text { patients }\end{array}$ & Main aim & Outcome & CRPT & Prognostic value $^{+}$ & $\begin{array}{l}\text { Cut-off } \\
\mathrm{mg} \cdot \mathrm{L}^{-1}\end{array}$ \\
\hline ZhANG [41] & 2011 & $\begin{array}{l}\text { Prospective, } \\
\text { single-institution; } \\
\text { 2001-2003; } \\
\text { the Netherlands }\end{array}$ & 405 & $\begin{array}{l}\text { COPD; } \\
\text { stable }\end{array}$ & $\begin{array}{l}\text { To quantify the effect of } \\
\text { cardiovascular determinants } \\
\text { on mortality in patients with a } \\
\text { diagnosis of COPD }\end{array}$ & $\begin{array}{l}\text { Late } \\
\text { mortality }\end{array}$ & Alone & $\begin{array}{c}\text { Multivariate; } 1.78 \text { (1.15-2.82); } \\
\text { age, FEV1, angina pectoris }\end{array}$ & 3 \\
\hline HøISETH [42] & 2012 & $\begin{array}{l}\text { Prospective, } \\
\text { single-institution; } \\
\text { 2005-2006; } \\
\text { Norway }\end{array}$ & 99 & $\begin{array}{l}\text { COPD; } \\
\text { acute }\end{array}$ & $\begin{array}{l}\text { To test the hypothesis that } \\
\mathrm{N} \text {-terminal pro-brain } \\
\text { natriuretic peptide } \\
\text { independently predicts } \\
\text { long-term mortality following } \\
\text { acute exacerbations of COPD }\end{array}$ & $\begin{array}{l}\text { Late } \\
\text { mortality }\end{array}$ & Alone & $\begin{array}{l}\text { Multivariate; } 2.4(1.7-3.2) \\
\text { age, sex, comorbidity, BMI, } \\
\mathrm{SaO}_{2} \text {, troponin level }\end{array}$ & 50 \\
\hline Deng [43] & 2014 & $\begin{array}{l}\text { Prospective, } \\
\text { single-institution; } \\
\text { 2009-2012; } \\
\text { China }\end{array}$ & 116 & $\begin{array}{l}\text { COPD; } \\
\text { stable }\end{array}$ & $\begin{array}{l}\text { To evaluate whether circulating } \\
\text { CRP levels are a biomarker of } \\
\text { systemic inflammation and a } \\
\text { significant predictor of future } \\
\text { COPD outcome }\end{array}$ & $\begin{array}{l}\text { Late } \\
\text { mortality }\end{array}$ & Alone & Univariate; 2.71 (1.05-6.99) & 3 \\
\hline Moberg [44] & 2014 & $\begin{array}{l}\text { Prospective, } \\
\text { single-institution; } \\
\text { 2005-2011; } \\
\text { Denmark }\end{array}$ & 423 & $\begin{array}{l}\text { COPD; } \\
\text { stable }\end{array}$ & $\begin{array}{l}\text { To investigate if leukocytes, CRP } \\
\text { and vitamin D are } \\
\text { independent predictors of } \\
\text { mortality and hospitalisation } \\
\text { after adjusting for disease } \\
\text { severity with an integrative } \\
\text { index (i-BODE index) }\end{array}$ & $\begin{array}{l}\text { Late } \\
\text { mortality }\end{array}$ & Alone & $\begin{array}{l}\text { Multivariate; } 1.50(1.07-2.10) ; \\
\text { age, sex, i-BODE index }\end{array}$ & 10 \\
\hline CANo [45] & 2014 & $\begin{array}{l}\text { Prospective, } \\
\text { multicentric; } \\
\text { recruitment period } \\
1 \text { year; } \\
\text { France }\end{array}$ & 637 & $\begin{array}{l}\text { COPD; } \\
\text { stable }\end{array}$ & $\begin{array}{l}\text { To investigate predictors of } \\
\text { long-term survival, including } \\
\text { respiratory, nutritional and } \\
\text { inflammatory dimensions, in } \\
\text { a prospective cohort of } \\
\text { home-treated patients with } \\
\text { chronic respiratory failure }\end{array}$ & $\begin{array}{l}\text { Late } \\
\text { mortality }\end{array}$ & Alone & $\begin{array}{l}\text { Multivariate; } 1.51(1.13-2.02) ; \\
\text { age, } P_{\mathrm{aO}_{2}}, P_{\mathrm{aCO}_{2}}, \mathrm{BMI} \\
\text { FEV } 1 / \mathrm{FVC}, 6 \mathrm{MWT} \text { distance, } \\
\text { transthyretin }\end{array}$ & 5 \\
\hline FoRD [46] & 2015 & $\begin{array}{l}\text { Prospective, } \\
\text { multicentric; } \\
\text { 1988-1994; } \\
\text { USA }\end{array}$ & 1144 & $\begin{array}{l}\text { COPD; } \\
\text { stable }\end{array}$ & $\begin{array}{l}\text { To examine the association } \\
\text { between elevated } \\
\text { inflammatory marker count } \\
\text { (white blood cell count, CRP } \\
\text { and fibrinogen) on all-cause } \\
\text { mortality in a national sample } \\
\text { of US adults with obstructive } \\
\text { lung function }\end{array}$ & $\begin{array}{l}\text { Late } \\
\text { mortality }\end{array}$ & $\begin{array}{l}\text { Alone/ } \\
\text { combined } \\
\text { (white blood } \\
\text { cell count, } \\
\text { fibrinogen) }\end{array}$ & $\begin{array}{l}\text { Univariate for CRP alone; } \\
1.26 \text { (0.95-1.66)/multivariate } \\
\text { for CRP combined; } \\
2.08 \text { (1.29-3.37); age, sex, } \\
\text { race, education, smoking } \\
\text { status, comorbidity, physical } \\
\text { activity, alcohol use, BMI, } \\
\text { FEV } / \text { FVC, blood pressure, } \\
\text { cholesterol level, urinary } \\
\text { albumin/creatinine ratio, } \\
\text { cancer history }\end{array}$ & 3 \\
\hline
\end{tabular}




\begin{tabular}{|c|c|c|c|c|c|c|c|c|c|}
\hline $\begin{array}{l}\text { First author } \\
\text { [ref.] }\end{array}$ & Year & $\begin{array}{l}\text { Study design; } \\
\text { recruitment period; } \\
\text { location }\end{array}$ & $\begin{array}{c}\text { Patients } \\
n\end{array}$ & $\begin{array}{c}\text { Type of } \\
\text { patients }\end{array}$ & Main aim & Outcome & CRPI & Prognostic value $^{+}$ & $\begin{array}{l}\text { Cut-off } \\
\mathrm{mg} \cdot \mathrm{L}^{-1}\end{array}$ \\
\hline KLeber [47] & 2015 & $\begin{array}{l}\text { Prospective, } \\
\text { single-institution; } \\
\text { 1997-2001; } \\
\text { Germany }\end{array}$ & 777 & $\begin{array}{l}\text { Smokers; } \\
\text { stable }\end{array}$ & $\begin{array}{l}\text { To characterise the diagnostic } \\
\text { value of two independent risk } \\
\text { factors for cardiovascular } \\
\text { events (high-sensitivity CRP } \\
\text { and lipoprotein-associated } \\
\text { phospholipase A2), which } \\
\text { provide information on } \\
\text { inflammation and plaque } \\
\text { stability in active smokers } \\
\text { and never-smokers of the } \\
\text { Ludwigshafen Risk and } \\
\text { Cardiovascular Health } \\
\text { (LURIC) study }\end{array}$ & $\begin{array}{c}\text { Late } \\
\text { mortality }\end{array}$ & $\begin{array}{l}\text { Combined } \\
\text { (lipoprotein- } \\
\text { associated } \\
\text { phospholipase } \\
\text { A2) }\end{array}$ & $\begin{array}{l}\text { Multivariate; } 1.94 \text { (1.10-3.45); } \\
\text { age, sex, comorbidity BMI, } \\
\text { cholesterol level, } \\
\text { triglycerides }\end{array}$ & 3.6 \\
\hline BLumenthal [48] & 2015 & $\begin{array}{l}\text { Prospective } \\
\text { (randomised } \\
\text { controlled trial), } \\
\text { multicentric; } \\
\text { 2009-2014; } \\
\text { USA }\end{array}$ & 326 & $\begin{array}{l}\text { COPD; } \\
\text { stable }\end{array}$ & $\begin{array}{l}\text { To examine the prognostic value } \\
\text { of select biobehavioural } \\
\text { factors in patients with COPD } \\
\text { in a secondary analysis of } \\
\text { participants from the } \\
\text { INSPIRE-II trial }\end{array}$ & $\begin{array}{l}\text { Late } \\
\text { mortality }\end{array}$ & Alone & $\begin{array}{l}\text { Multivariate; } 2.25 \text { (1.02-4.96); } \\
\text { age, Charlson score, COPD } \\
\text { duration, GOLD, } \\
\text { corticosteroids, coping } \\
\text { skills training }\end{array}$ & $\begin{array}{c}\text { Not } \\
\text { reported }\end{array}$ \\
\hline LoPRINZI [49] & 2016 & $\begin{array}{l}\text { Retrospective, } \\
\text { single-institution; } \\
\text { 2003-2006; } \\
\text { USA }\end{array}$ & 385 & $\begin{array}{l}\text { COPD; } \\
\text { stable }\end{array}$ & $\begin{array}{l}\text { To examine the association } \\
\text { between objectively measured } \\
\text { physical activity and all-cause } \\
\text { mortality among a national } \\
\text { sample of COPD patients, } \\
\text { with stratification by } \\
\text { inflammatory status }\end{array}$ & $\begin{array}{c}\text { Late } \\
\text { mortality }\end{array}$ & Alone & $\begin{array}{l}\text { Multivariate; } 1.33 \text { (1.02-1.72); } \\
\text { age, sex, comorbidity, race, } \\
\text { BMI, physical activity, } \\
\text { poverty/income ratio, } \\
\text { cotinine }\end{array}$ & $\begin{array}{c}\text { Not } \\
\text { reported }\end{array}$ \\
\hline SALTÜRK [50] & 2015 & $\begin{array}{l}\text { Retrospective, } \\
\text { single-institution; } \\
\text { 2013-2014; } \\
\text { Turkey }\end{array}$ & 647 & $\begin{array}{l}\text { COPD; } \\
\text { acute }\end{array}$ & $\begin{array}{l}\text { To assess whether eosinophilic } \\
\text { COPD exacerbations have } \\
\text { better outcomes than } \\
\text { noneosinophilic COPD } \\
\text { exacerbations in the intensive } \\
\text { care unit }\end{array}$ & $\begin{array}{c}\text { Early } \\
\text { mortality }\end{array}$ & Alone & $\begin{array}{l}\text { Multivariate; } 1.78 \text { (1.01-3.14); } \\
\text { age, sex, BMI, NLR, } \\
\text { eosinophilia, invasive } \\
\text { mechanical ventilation, } \\
\text { noninvasive mechanical } \\
\text { ventilation, APACHE II } \\
\text { score, septic shock, } \\
\text { resistant pathogen }\end{array}$ & 500 \\
\hline MURPHY [51] & 2010 & $\begin{array}{l}\text { Retrospective, } \\
\text { single-institution; } \\
\text { 2004-2007; } \\
\text { UK }\end{array}$ & 60 & $\begin{array}{l}\text { COPD; } \\
\text { acute }\end{array}$ & $\begin{array}{l}\text { To determine if routine clinical } \\
\text { assessment could reliably } \\
\text { predict in-hospital death in } \\
\text { patients admitted with acute } \\
\text { exacerbation of COPD }\end{array}$ & $\begin{array}{c}\text { Early } \\
\text { mortality }\end{array}$ & Alone & $\begin{array}{l}\text { Multivariate; } 1.22(0.95-1.14) \\
\text { Charlson score, } \mathrm{pH} \text {, urea }\end{array}$ & $\begin{array}{c}\text { Not } \\
\text { reported }\end{array}$ \\
\hline
\end{tabular}


TABLE 1 Continued

\begin{tabular}{|c|c|c|c|c|c|c|c|c|c|}
\hline $\begin{array}{l}\text { First author } \\
\text { [ref.] }\end{array}$ & Year & $\begin{array}{l}\text { Study design; } \\
\text { recruitment period; } \\
\text { location }\end{array}$ & $\begin{array}{l}\text { Patients } \\
\text { n }\end{array}$ & $\begin{array}{c}\text { Type of } \\
\text { patients }\end{array}$ & Main aim & Outcome & CRP & Prognostic value $^{+}$ & $\begin{array}{l}\text { Cut-off } \\
\mathrm{mg} \cdot \mathrm{L}^{-1}\end{array}$ \\
\hline Zнао [52] & 2014 & $\begin{array}{l}\text { Prospective, } \\
\text { single-institution; } \\
\text { 2010-2011; } \\
\text { China }\end{array}$ & 159 & $\begin{array}{l}\text { COPD; } \\
\text { acute }\end{array}$ & $\begin{array}{l}\text { To investigate the COPD } \\
\text { assessment test, serum } \\
\text { copeptin, procalcitonin and } \\
\text { CRP levels as potential } \\
\text { predictive factors for } \\
\text { recurrence of acute } \\
\text { exacerbation and all-cause } \\
\text { mortality in } 6 \text { months in } \\
\text { COPD inpatients }\end{array}$ & $\begin{array}{c}\text { Early } \\
\text { mortality }\end{array}$ & Alone & Univariate; 0.90 (0.82-1.01) & $\begin{array}{l}\text { Not } \\
\text { reported }\end{array}$ \\
\hline StoLz [53] & 2008 & $\begin{array}{l}\text { Prospective, } \\
\text { single-institution; } \\
\text { 2003-2005; } \\
\text { Germany }\end{array}$ & 167 & $\begin{array}{l}\text { COPD; } \\
\text { acute }\end{array}$ & $\begin{array}{l}\text { To investigate whether plasma } \\
\text { pro-endothelin-1 and/or } \\
\text { pro-adrenomedullin on } \\
\text { admission to the hospital for } \\
\text { acute exacerbation predict } \\
\text { survival in patients with COPD }\end{array}$ & $\begin{array}{l}\text { Early } \\
\text { Survival }\end{array}$ & Alone & $\begin{array}{l}\text { Multivariate; } 1.02 \text { (0.92-1.13); } \\
\text { age, Charlson score, } \mathrm{PaO}_{2}, \\
\mathrm{PaCO}_{2} \text {, pro-adrenomedullin, } \\
\text { endothelin-1, BMI, FEV1, } \\
\text { leukocyte counts, } \\
\text { procalcitonin, pulmonary } \\
\text { arterial hypertension }\end{array}$ & $\begin{array}{l}\text { Not } \\
\text { reported }\end{array}$ \\
\hline Duman [54] & 2015 & $\begin{array}{l}\text { Retrospective, } \\
\text { single-Institution; } \\
2014 \text { (1 year); } \\
\text { Turkey }\end{array}$ & 1704 & $\begin{array}{l}\text { COPD; } \\
\text { acute }\end{array}$ & $\begin{array}{l}\text { To evaluate mortality and } \\
\text { outcomes of eosinophilic and } \\
\text { noneosinophilic COPD } \\
\text { exacerbations, and identify } \\
\text { new biomarkers that predict } \\
\text { survival }\end{array}$ & $\begin{array}{l}\text { Early } \\
\text { mortality }\end{array}$ & Alone & $\begin{array}{l}\text { Multivariate; } 1.32 \text { (1.01-1.71); } \\
\text { cardiovascular disease, } \\
\text { corticosteroids, length of } \\
\text { stay, readmission, NLR }\end{array}$ & 19 \\
\hline TofAN [55] & 2012 & $\begin{array}{l}\text { Prospective, } \\
\text { single-institution; } \\
\text { 1999-2010; } \\
\text { Iran }\end{array}$ & 60 & $\begin{array}{l}\text { COPD; } \\
\text { acute }\end{array}$ & $\begin{array}{l}\text { To assess the clinical utility of } \\
\text { serum high-sensitivity CRP at } \\
\text { admission in predicting } \\
\text { outcome in hospitalised } \\
\text { patients with acute } \\
\text { exacerbation COPD }\end{array}$ & $\begin{array}{l}\text { Early } \\
\text { mortality }\end{array}$ & Alone & Univariate; 4.04 (1.32-12.33) & 100 \\
\hline ZhANG [56] & 2014 & $\begin{array}{l}\text { Prospective, } \\
\text { single-institution; } \\
\text { 2007-2012; } \\
\text { China }\end{array}$ & 378 & $\begin{array}{l}\text { COPD; } \\
\text { acute }\end{array}$ & $\begin{array}{l}\text { To assess the association of } \\
\text { high-sensitivity CRP with } \\
\text { in-hospital outcomes in } \\
\text { patients with COPD } \\
\text { undergoing percutaneous } \\
\text { coronary intervention }\end{array}$ & $\begin{array}{l}\text { Early } \\
\text { mortality }\end{array}$ & Alone & $\begin{array}{l}\text { Multivariate; } 1.78 \text { (1.15-2.82); } \\
\text { FEV1, left ventricular } \\
\text { ejection fraction, } \\
\text { three-vessel disease, } \\
\beta \text {-blocker use }\end{array}$ & 3 \\
\hline ANDREASSEN [57] & 2014 & $\begin{array}{l}\text { Retrospective, } \\
\text { multicentric; } \\
\text { 2005; } \\
\text { Norway/Sweden }\end{array}$ & 731 & $\begin{array}{l}\text { COPD; } \\
\text { acute }\end{array}$ & $\begin{array}{l}\text { To find the proportion of } \\
\text { patients with pneumonia } \\
\text { among admissions due to } \\
\text { acute exacerbations COPD } \\
\text { and whether pneumonia has } \\
\text { an impact on the length of } \\
\text { stay, usage of noninvasive } \\
\text { ventilation or in-hospital } \\
\text { mortality }\end{array}$ & $\begin{array}{l}\text { Early } \\
\text { mortality }\end{array}$ & Alone & $\begin{array}{l}\text { Multivariate; } 0.71 \text { (0.28-1.82); } \\
\text { age, sex, GOLD }\end{array}$ & 40 \\
\hline
\end{tabular}




\begin{tabular}{|c|c|c|c|c|c|c|c|c|c|}
\hline $\begin{array}{l}\text { First author } \\
\text { [ref.] }\end{array}$ & Year & $\begin{array}{l}\text { Study design; } \\
\text { recruitment period; } \\
\text { location }\end{array}$ & $\begin{array}{c}\text { Patients } \\
\text { n }\end{array}$ & $\begin{array}{c}\text { Type of } \\
\text { patients }\end{array}$ & Main aim & Outcome & CRPI & Prognostic value $^{+}$ & $\begin{array}{l}\text { Cut-off } \\
\mathrm{mg} \cdot \mathrm{L}^{-1}\end{array}$ \\
\hline GuertLer [58] & 2011 & $\begin{array}{l}\text { Prospective, } \\
\text { multicentric; } \\
\text { 2006-2008; } \\
\text { Switzerland }\end{array}$ & 877 & $\begin{array}{l}\text { COPD; } \\
\text { acute }\end{array}$ & $\begin{array}{l}\text { To investigate the long-term } \\
\text { prognostic performance of } \\
\text { the Pneumonia Severity Index } \\
\text { score and the association of } \\
\text { clinical parameters and } \\
\text { different blood biomarkers } \\
\text { with long-term mortality rate } \\
\text { in a large cohort of patients } \\
\text { with community-acquired } \\
\text { pneumonia }\end{array}$ & $\begin{array}{c}\text { Early } \\
\text { mortality }\end{array}$ & Alone & $\begin{array}{l}\text { Multivariate; } 0.3 \text { (0.2-0.5); } \\
\text { age, sex, comorbidity, } \\
\text { temperature, chills, } \\
\text { pro-adrenomedullin }\end{array}$ & $\begin{array}{l}\text { Not } \\
\text { reported } \\
\text { (fourth } \\
\text { quartile) }\end{array}$ \\
\hline MoghBELI [59] & 2005 & $\begin{array}{l}\text { Prospective, } \\
\text { multicentric; } \\
\text { 1997-1999; } \\
\text { USA }\end{array}$ & 1862 & $\begin{array}{l}\text { Smokers; } \\
\text { acute }\end{array}$ & $\begin{array}{l}\text { To investigate the role of } \\
\text { inflammation, as measured } \\
\text { by high-sensitivity CRP levels, } \\
\text { in cardiovascular risk in } \\
\text { smokers who have acute } \\
\text { coronary syndrome }\end{array}$ & $\begin{array}{c}\text { Early } \\
\text { mortality }\end{array}$ & Alone & $\begin{array}{l}\text { Multivariate; } 2.60(1.42-4.79) \\
\text { age, comorbidity, previous } \\
\text { treatment, troponin level }\end{array}$ & 15 \\
\hline HAJa Mydin [60] & 2013 & $\begin{array}{l}\text { Prospective, } \\
\text { single-institution; } \\
\text { 2009-2010; } \\
23 \text { months; } \\
\text { UK }\end{array}$ & 65 & $\begin{array}{l}\text { COPD; } \\
\text { acute }\end{array}$ & $\begin{array}{l}\text { To identify factors associated } \\
\text { with inpatient mortality from } \\
\text { hypercapnic respiratory } \\
\text { failure with respiratory } \\
\text { acidosis due to COPD }\end{array}$ & $\begin{array}{c}\text { Early } \\
\text { mortality }\end{array}$ & Alone & Univariate; 3.43 (0.38-30.55) & 3 \\
\hline
\end{tabular}

HR: hazard ratio; $\mathrm{BMI}$ : body mass index; $\mathrm{FEV}_{1}$ : forced expiratory volume in $1 \mathrm{~s}$; BODE: BMI, airflow obstruction, dyspnoea and exercise capacity; SaO ${ }_{2}$ : arterial oxygen saturation; PaO ${ }_{2}$ : arterial oxygen tension; $\mathrm{PaCO}_{2}$ : arterial carbon dioxide tension; FVC: forced vital capacity; 6MWT: 6-min walk test; GOLD: Global Initiative for Chronic Obstructive Lung Disease; NLR: neutrophil/lymphocyte ratio. ${ }^{\#}$ : COPD or smokers; stable or acute; ${ }^{\text {I: }}$ alone or combined; ${ }^{+}$: univariate or multivariate; HR $(95 \% \mathrm{CI})$; adjusted covariables. 
different subsets of COPD patients were identified: patients with stable COPD $(n=11081)$ and those with acute exacerbations $(n=6809)$. Of these 26 articles, 15 studies with 11180 patients focused on late mortality [35-49] and 11 studies with 6710 patients focused on early mortality [50-60]. Patients with acute COPD were enrolled in one [42] and all articles focusing on late and early mortality, respectively. The CRP cut-off values were based on study-specific categories reported in all studies. The CRP level was combined with another factor in two studies [46, 47]. Adjusted mortality risks estimates were available for 23 studies. The most common variables accounted for adjusting multivariable analyses in these studies were: age ( $\mathrm{n}=18$ articles) [35-39, 41, 42, 44-50, 53, 57-59], comorbidities ( $\mathrm{n}=15$ articles) [37-39, 41, 42, 46-49, 51, $53,54,56,58,59]$, COPD severity ( $\mathrm{n}=10$ articles) $[36,37,41,42,45,46,48,53,56,57]$, sex ( $\mathrm{n}=9$ articles) $[36,37,42,44,46,47,50,57,58]$, body mass index ( $\mathrm{n}=8$ articles) $[36,42,45-47,49,50,53]$ and smoking ( $\mathrm{n}=6$ articles) [35-39, 46].

\section{Meta-analysis on late mortality}

The pooled analysis on 15 late mortality studies including 11180 patients showed a significant positive association between elevated baseline CRP levels and increase in late mortality (HR 1.53, 95\% CI 1.32-1.77), with a moderate level of heterogeneity $\left(I^{2}=68.7 \%, \mathrm{p}<0.001\right)$ (figure 2 ). The results were not driven by any single study as the exclusion of each study from the meta-analysis did not materially change the summary estimate.

Subgroup analysis was performed by stratifying for geographic areas and CRP cut-off values. No heterogeneity was observed across the strata considered (table 2). However, we found a higher risk for late mortality in four studies that used a study-specific CRP cut-off value of $3 \mathrm{mg} \cdot \mathrm{L}^{-1}$ (HR 1.61,95\% CI 1.12-2.30) and in two studies focusing on Asiatic populations (HR 3.51, 95\% CI 1.69-7.31). The presence of publication bias was evidenced by visual inspection of the funnel plot and by using Egger's $(p=0.01)$ and Begg's ( $p=0.006)$ tests (online supplementary figure S1a). Adjustment for publication bias according to the "trim and fill" method resulted in HR 1.33 (95\% CI 1.10-1.60), with six studies imputed (online supplementary figure S1b).

\section{Meta-analysis on early mortality}

According to the relationship between CRP level at baseline and early mortality, eight studies (based on 4943 patients) and three studies (based on 1767 patients) reported a relative risk $>1$ and $<1$, respectively. The pooled analysis revealed a nonsignificant association between CRP levels and early mortality (relative risk 1.15, 95\% CI 0.94-1.42), with a high level of heterogeneity $\left(I^{2}=86.7 \%, \mathrm{p}<0.001\right)$ (figure 3 ).

No evidence of publication bias was reported by visual inspection of the funnel plot and by using Egger's $(\mathrm{p}=0.53$ ) and Begg's $(\mathrm{p}=0.88)$ tests (online supplementary figure $\mathrm{S} 2$ ).

\begin{tabular}{|c|c|c|c|}
\hline First author [ref.] & & $\begin{array}{c}\text { Hazard ratio } \\
(95 \% \mathrm{Cl})\end{array}$ & $\begin{array}{c}\text { Weight } \\
\%\end{array}$ \\
\hline KULLER [35] & $=$ & $2.80(1.43-5.50)$ & 3.39 \\
\hline MAN [36] & - & $1.79(1.25-2.56)$ & 6.96 \\
\hline DAHL [37] & + & $1.40(1.09-1.79)$ & 8.90 \\
\hline DE TORRES [38] & + & $1.00(0.82-1.22)$ & 9.75 \\
\hline MeHROtRa [39] & - & $1.12(0.93-1.35)$ & 10.00 \\
\hline LIU [40] & $\longrightarrow$ & $5.15(1.63-16.31)$ & 1.43 \\
\hline ZHANG [41] & $\frac{1}{4}$ & $1.48(1.28-1.71)$ & 10.64 \\
\hline HøISETH [42] & $\rightarrow$ & $2.40(1.75-3.29)$ & 7.66 \\
\hline CANO [45] & - & $1.51(1.13-2.02)$ & 8.11 \\
\hline DeNG [43] & $\longrightarrow$ & $2.71(1.05-7.00)$ & 2.00 \\
\hline MOBERg [44] & $-\frac{1}{1}$ & $1.50(1.07-2.10)$ & 7.31 \\
\hline FoRD [46] & 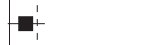 & $1.26(0.95-1.67)$ & 8.31 \\
\hline KLEBER [47] & \begin{tabular}{c:c}
-1 \\
\hdashline-
\end{tabular} & $1.94(1.10-3.44)$ & 4.25 \\
\hline BLUMENTHAL [48] & \begin{tabular}{l:}
$1:$ \\
\hdashline-1
\end{tabular} & $2.25(1.02-4.96)$ & 2.68 \\
\hline LOPRINZI [49] & -1 & $1.33(1.02-1.73)$ & 8.63 \\
\hline Overall $(/ 2=68.7 \%, p=0.000)$ & 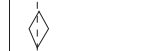 & $1.53(1.32-1.77)$ & 100.00 \\
\hline
\end{tabular}

FIGURE 2 Forest plot for the association between C-reactive protein level and late mortality in chronic obstructive pulmonary disease patients. Studies are listed in chronological order (see table 1 for full study details). 


\begin{tabular}{|c|c|c|c|c|}
\hline & Studies $\mathbf{n}$ & HR $(95 \% \mathrm{CI})$ & $I^{2} \%$ & Pheterogeneity $_{\text {-value }}$ \\
\hline \multicolumn{4}{|l|}{ Geographic area } & \\
\hline Europe & 6 & $1.61(1.38-1.87)$ & 46.8 & 0.06 \\
\hline USA and Canada & 5 & $1.34(1.12-1.61)$ & 46.2 & \\
\hline Europe and USA (multicentric) & 2 & $1.59(0.58-4.33)$ & 87.8 & \\
\hline China & 2 & $3.51(1.69-7.31)$ & 0 & \\
\hline \multicolumn{5}{|c|}{ Study-specific CRP cut-off $3 \mathrm{mg} \cdot \mathrm{L}^{-1^{\#}}$} \\
\hline$\leqslant 3 \mathrm{mg} \cdot \mathrm{L}^{-1}$ & 4 & $1.61(1.12-2.30)$ & 58.9 & 0.85 \\
\hline$>3 \mathrm{mg} \cdot \mathrm{L}^{-1}$ & 9 & $1.55(1.29-1.85)$ & 70.7 & \\
\hline \multicolumn{5}{|c|}{ Study-specific CRP cut-off $5 \mathrm{mg} \cdot \mathrm{L}^{-1^{\#}}$} \\
\hline$\leqslant 5 \mathrm{mg} \cdot \mathrm{L}^{-1}$ & 6 & $1.55(1.25-1.93)$ & 40.5 & 0.93 \\
\hline$>5 \mathrm{mg} \cdot \mathrm{L}^{-1}$ & 7 & $1.53(1.23-1.91)$ & 77.0 & \\
\hline
\end{tabular}

\#: two studies did not report the cut-off value $[35,39]$.

\section{Discussion}

The prognostic role of baseline CRP level has been well established in the setting of cardiovascular disease [61, 62] as well as in cancer patients [17, 63], but the effect of CRP on COPD mortality is still unclear, and the association between baseline CRP levels and survival remains controversial in COPD patients [64, 65]. In this clinical setting, it has been suggested that elevated levels of CRP are related to the occurrence of cardiovascular events, rather than mortality from COPD itself [66]. However, other authors reported that CRP increase is secondary to serum concentration of other pro-inflammatory cytokines (TNF- $\alpha$, IL-6, IL-8 or fibrinogen) [39].

The pooled estimates of the present meta-analysis revealed a consistent and significant association between elevated baseline CRP levels and mortality in patients with COPD. The only prior meta-analysis on this subject included four papers and 460 patients, showing a nonsignificant relative risk of 1.72 (95\% CI 0.75-3.95) [67]. Although these data may appear controversial, our study pooled the results of 15 studies and 11180 patients, thereby reaching solid statistical significance.

Two different mechanisms have been proposed to explain the association between CRP and COPD. The first hypothesis is related to the effect of lung inflammation itself in COPD patients. It is well known that prolonged exposure to cigarettes leads to lung injury and inflammation [9]. Once this process starts, lung inflammation persists even after smoking cessation [68], thus resulting in an exponential systemic reaction, related to the severity of COPD [69]. However, the parallel activation of systemic inflammation maintains

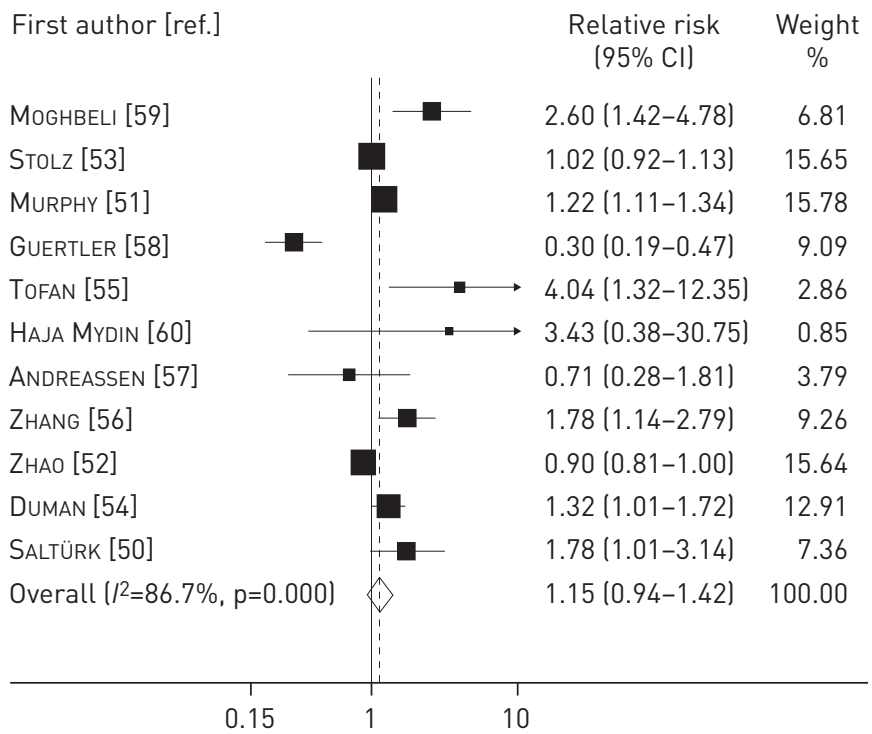

FIGURE 3 Forest plot for the association between C-reactive protein level and early mortality in chronic obstructive pulmonary disease patients. Studies are listed in chronological order (see table 1 for full study details). 
and increases the local airway inflammation $[68,69]$, leading to COPD progression. The second hypothesis is linked to systemic inflammatory factors (e.g. CRP, fibrinogen, IL-6, leukocytes and platelets) that are major mediators for atherosclerosis and cardiovascular diseases. In this setting, Suwa et al. [70] studied the effect of air pollution and reported that the concentration of alveolar macrophages containing particulate matter was directly proportional to the extent of atherosclerosis. Similarly, the Tucson Epidemiologic Study of Airways Obstructive Disease reported that only $8 \%$ of patients with COPD died because of lung disease, whereas the majority of patients died either from cardiovascular diseases or cancer [71]. Furthermore, although respiratory failure represents a significant cause of mortality in severe COPD, cardiovascular events and lung cancer still account for a large proportion of deaths even in this group of patients [72]. The association with heart disease is further supported by other studies reporting increased levels of N-terminal pro-brain natriuretic peptide and troponin- $\mathrm{T}$ in such patients [73]. Thus, it is not surprising that CRP (related to immune status imbalance) could be used as a prognostic marker in COPD, as inflammation itself may lead both to lung damage persistence and cardiovascular events in these patients. However, we are aware that other possible mechanisms may be related to CRP increase in COPD patients (e.g. associated comorbidities, inactivity, etc.). Further data are needed to better investigate these associations.

An interesting issue that has not been properly explored in the literature is the optimal CRP cut-off value to predict the outcome in COPD patients. Although the discriminant values of other biomarkers have been investigated [74], no studies are available in the setting of CRP levels. Our analysis indicated different cut-off points among each study, ranging from 3 to $50 \mathrm{mg} \cdot \mathrm{L}^{-1}$ and from 3 to $500 \mathrm{mg} \cdot \mathrm{L}^{-1}$ in those focusing on late and early mortality, respectively. Considering late mortality, we performed a subgroup analysis according to different CRP cut-off points $\left(3\right.$ and $\left.5 \mathrm{mg} \cdot \mathrm{L}^{-1}\right)$ in order to obtain an optimal stratification. We demonstrated a better discrimination for those studies adopting a cut-off point $\leqslant 3 \mathrm{mg} \cdot \mathrm{L}^{-1}$. These data are similar to the those reported in a previous meta-analysis on early-stage lung cancer [17], confirming an interesting dual role of CRP (a mirror of the underlying systemic inflammation) in predicting the prognosis of two different diseases (COPD and lung cancer) sharing the same risk factors (smoking, occupational exposures and chronic inflammation). However, the data on this issue are limited and we advocate the need for further studies to better investigate this association.

Considering the immune hyperactivity that characterises COPD, other prognostic biomarkers have already been evaluated in the literature. Reportedly, fibrinogen has been chosen by the Food and Drug Administration as the "gold standard" to assess systemic inflammation in COPD. In particular, fibrinogen levels $>350 \mathrm{mg} \cdot \mathrm{dL}^{-1}$ may help clinicians to identify COPD individuals at an increased risk of exacerbations and death [75]. Similarly, adrenomedullin [76] and copeptin [27, 52, 77] (both biomarkers reflecting different pathobiological pathways) have been recently reported as COPD prognostic biomarkers. In this setting, the use of drugs to modulate systemic inflammation (and also biomarkers) is an interesting issue that should be better assessed. Pharmacotherapies with bronchodilators such as $\beta_{2}$-agonists, anticholinergics and theophylline have been evaluated, although the effect of such local drugs on CRP level reduction is controversial [78]. However, the use of statins in such patients seems promising. In addition to their lipid-lowering effect, statins have anti-inflammatory and immunomodulating properties, reducing the levels of inflammatory markers such as CRP [79]. This effect was further confirmed in a randomised controlled trial performed by LEE et al. [80] reporting that pravastatin treatment significantly decreased CRP and IL-6 levels compared with placebo, and that the improvement of exercise tolerance was greater in those with a greater decrease of CRP levels and higher baseline CRP levels. Similarly, other authors suggest that patients with COPD treated with statins have an advantage in terms of morbidity and mortality, by reducing the rate of mortality from pneumonia or infective exacerbations, by slowing the decline in FEV1, and by improving exercise tolerance [81]. It is noteworthy that the Rotterdam Study recently reported long-term statin use was associated with a $78 \%$ reduction in mortality if the CRP level was $>3 \mathrm{mg} \cdot \mathrm{L}^{-1}$ versus a nonsignificant $21 \%$ reduction if the CRP level was $\leqslant 3 \mathrm{mg} \cdot \mathrm{L}^{-1}$ [25]. This interaction is of particular interest if one considers that the baseline CRP level (e.g. using a cut-off of $3 \mathrm{mg} \cdot \mathrm{L}^{-1}$ ) could be used to stratify COPD patients in low- and high-risk subsets. Such stratification might be useful to select which patients would benefit from immunomodulating therapies. In this setting, the use of widely available drugs such as aspirin seems beneficial, as reported in a recent meta-analysis showing a reduction of all-cause mortality in patients with COPD receiving anti-platelet treatment (relative risk 0.81 , 95\% CI 0.75-0.88) [82]. Probably, the preventive effect of aspirin may be linked to the reduction of both systemic inflammation and atherosclerosis in such patients. Further well-designed prospective studies are needed to better clarify the role of these combined therapies.

Another matter of debate is the prediction of early outcome in COPD patients, especially in those with acute exacerbations. This issue is of interest to physicians in daily clinical practice. Studies have shown that the early outcome of COPD is related mainly to exacerbations due to infection, either viral or bacterial, or both; these acute events are associated with high economic costs and accelerated lung function decline 
[83]. It is widely established that CRP is a useful and sensitive indicator of infections and acute exacerbations in patients with COPD [84]. Some authors have reported that high CRP levels are associated with the need for intensive care unit transfer, intubation or mechanical ventilation, congestive heart failure onset, and higher in-hospital and after-discharge mortality [85]. Based on our pooled analysis on early mortality, we evidenced a weak association only between CRP and short-term outcome in COPD. However, the results on this issue may be influenced by the high heterogeneity reported in the 11 analysed studies. We advocate for further studies to better clarify these associations.

\section{Study limitations and strengths}

This meta-analysis has some limitations. First, although the majority of studies were prospective, no study was randomised. Thus, biases in treatment selection and covariate distribution cannot be excluded, resulting in difficult estimation of the real prognostic role of CRP. Furthermore, given the lack of data on the causes of mortality, the real cause of death was not properly explored in this meta-analysis, leaving the possibility that CRP level could actually be associated with increased deaths due to comorbidities. Second, several articles reported different CRP cut-off values, thus making study comparison difficult. Third, these studies were heterogeneous in terms of geographic area, age, sex and type of COPD. Fourth, there was publication bias, but the "trim and fill" method confirmed the main result. Finally, although the main results focused on late mortality, the pooled analysis on early mortality revealed a high level of heterogeneity, probably because different events (all-cause mortality within 24 months, in-hospital complications and hospitalisation) were accounted for in those studies focusing on this outcome.

The main strength of our study is the combination of all published data up to now using a meta-analytic approach. Furthermore, the systematic review and meta-analysis was strictly performed according to the MOOSE guidelines.

\section{Conclusions}

Systemic inflammation plays an important role in COPD pathogenesis, disease progression and mortality. Although CRP is associated with several conditions related to the natural history of COPD, its level may also be used to assess the outcome in COPD patients. Based on our analysis, CRP levels could be used, in combination with other biochemical markers, to target preventive and therapeutic strategies in this disease. Further prospective studies should be performed to confirm the clinical value of CRP.

\section{References}

1 Halbert RJ, Natoli JL, Gano A, et al. Global burden of COPD: systematic review and meta-analysis. Eur Respir J 2006; 28: 523-532.

2 WHO. Chronic obstructive pulmonary disease (COPD). www.who.int/respiratory/copd/burden/en/ Date last accessed: July 5, 2016.

3 Shah T, Churpek MM, Perraillon MC, et al. Understanding why patients with COPD get readmitted: a large national study to delineate the Medicare population for the readmissions penalty expansion. Chest 2015; 147: 1219-1226.

4 Pasquale MK, Sun SX, Song F, et al. Impact of exacerbations on health care cost and resource utilization in chronic obstructive pulmonary disease patients with chronic bronchitis from a predominantly Medicare population. Int J Chron Obstruct Pulmon Dis 2012; 7: 757-764.

5 Miller JD, Foster T, Boulanger L, et al. Direct costs of COPD in the U.S.: an analysis of Medical Expenditure Panel Survey (MEPS) data. COPD 2005; 2: 311-318.

6 Vestbo J, Hurd SS, Agustí AG, et al. Global strategy for the diagnosis, management, and prevention of chronic obstructive pulmonary disease: GOLD executive summary. Am J Respir Crit Care Med 2013; 187: 347-365.

7 Mannino DM, Buist AS. Global burden of COPD: risk factors, prevalence, and future trends. Lancet 2007; 370: $765-773$.

8 Kohansal R, Martinez-Camblor P, Agusti A, et al. The natural history of chronic airflow obstruction revisited: an analysis of the Framingham offspring cohort. Am J Respir Crit Care Med 2009; 180: 3-10.

9 Hogg JC. Pathophysiology of airflow limitation in chronic obstructive pulmonary disease. Lancet 2004; 364: 709-721.

10 Danesh J, Collins R, Appleby P, et al. Association of fibrinogen, C-reactive protein, albumin, or leukocyte count with coronary heart disease: meta-analyses of prospective studies. JAMA 1998; 279: 1477-1482.

11 Gan WQ, Man SF, Senthilselvan A, et al. Association between chronic obstructive pulmonary disease and systemic inflammation: a systematic review and a metaanalysis. Thorax 2004; 59: 574-580.

12 Danesh J, Whincup P, Walker M, et al. Low grade inflammation and coronary heart disease: prospective study and updated meta-analyses. BMJ 2000; 321: 199-204.

13 Guo YZ, Pan L, Du CJ, et al. Association between C-reactive protein and risk of cancer: a meta-analysis of prospective cohort studies. Asian Pac J Cancer Prev 2013; 14: 243-248.

14 Vestbo J, Agusti A, Wouters EF, et al. Should we view chronic obstructive pulmonary disease differently after ECLIPSE? A clinical perspective from the study team. Am J Respir Crit Care Med 2014; 189: 1022-1030.

15 McShane LM, Altman DG, Sauerbrei W. Identification of clinically useful cancer prognostic factors: what are we missing? J Natl Cancer Inst 2005; 97: 1023-1025.

16 De Gruttola VG, Clax P, DeMets DL, et al. Considerations in the evaluation of surrogate endpoints in clinical trials. summary of a National Institutes of Health workshop. Control Clin Trials 2001; 22: 485-502. 
17 Leuzzi G, Galeone C, Gisabella M, et al. Baseline C-reactive protein level predicts survival of early-stage lung cancer: evidence from a systematic review and meta-analysis. Tumori 2016; 102: 441-449.

18 Tierney JF, Stewart LA, Ghersi D, et al. Practical methods for incorporating summary time-to-event data into meta-analysis. Trials 2007; 8: 16.

19 Altman DG, Bland JM. How to obtain the confidence interval from a P value. BMJ 2011; 343: d2090.

20 Je Y, Liu W, Giovannucci E. Coffee consumption and risk of colorectal cancer: a systematic review and meta-analysis of prospective cohort studies. Int J Cancer 2009; 124: 1662-1668.

21 Stroup DF, Berlin JA, Morton SC, et al. Meta-analysis of observational studies in epidemiology: a proposal for reporting. Meta-analysis Of Observational Studies in Epidemiology (MOOSE) group. JAMA 2000; 283: 2008-2012.

22 Duval S, Tweedie R. Trim and fill: a simple funnel-plot-based method of testing and adjusting for publication bias in meta-analysis. Biometrics 2000; 56: 455-463.

23 Bernasconi $\mathrm{M}$, Tamm M, Bingisser $\mathrm{R}$, et al. Midregional proatrial natriuretic peptide predicts survival in exacerbations of COPD. Chest 2011; 140: 91-99.

24 Man SF, Xing L, Connett JE, et al. Circulating fibronectin to C-reactive protein ratio and mortality: a biomarker in COPD? Eur Respir J 2008; 32: 1451-1457.

25 Lahousse L, Loth DW, Joos GF, et al. Statins, systemic inflammation and risk of death in COPD: the Rotterdam study. Pulm Pharmacol Ther 2013; 26: 212-217.

26 Parker DR, Liu J, Roberts MB, et al. Is inflammatory chronic obstructive pulmonary disease a coronary heart disease risk equivalent? A longitudinal analysis of the third National Health and Nutrition Examination Survey (NHANES III), 1988-1994. BMC Pulm Med 2014; 14: 195.

27 Müller B, Morgenthaler N, Stolz D, et al. Circulating levels of copeptin, a novel biomarker, in lower respiratory tract infections. Eur J Clin Invest 2007; 37: 145-152.

28 Müller B, Süess E, Schuetz P, et al. Circulating levels of pro-atrial natriuretic peptide in lower respiratory tract infections. J Intern Med 2006; 260: 568-576.

29 Miniati M, Monti S, Bottai M, et al. Prognostic value of C-reactive protein in chronic obstructive pulmonary disease. Intern Emerg Med 2011; 6: 423-430.

30 Lacoma A, Prat C, Andreo F, et al. Value of procalcitonin, C-reactive protein, and neopterin in exacerbations of chronic obstructive pulmonary disease. Int J Chron Obstruct Pulmon Dis 2011; 6: 157-169.

31 Cano NJ, Pichard C, Roth $\mathrm{H}$, et al. C-reactive protein and body mass index predict outcome in end-stage respiratory failure. Chest 2004; 126: 540-546.

32 Waschki B, Kirsten A, Holz O, et al. Physical activity is the strongest predictor of all-cause mortality in patients with COPD: a prospective cohort study. Chest 2011; 140: 331-342.

33 Celli BR, Locantore N, Yates J, et al. Inflammatory biomarkers improve clinical prediction of mortality in chronic obstructive pulmonary disease. Am J Respir Crit Care Med 2012; 185: 1065-1072.

34 Jin $\mathrm{Q}$, Chen $\mathrm{Y}$, Lou $\mathrm{Y}$, et al. Low Serum retinol-binding protein-4 levels in acute exacerbations of chronic obstructive pulmonary disease at intensive care unit admission is a predictor of mortality in elderly patients. J Inflamm (Lond) 2013; 10: 31.

35 Kuller LH, Tracy RP, Shaten J, et al. Relation of C-reactive protein and coronary heart disease in the MRFIT nested case-control study. Multiple Risk Factor Intervention Trial. Am J Epidemiol 1996; 144: 537-547.

36 Man SF, Connett JE, Anthonisen NR, et al. C-reactive protein and mortality in mild to moderate chronic obstructive pulmonary disease. Thorax 2006; 61: 849-853.

37 Dahl M, Vestbo J, Lange P, et al. C-reactive protein as a predictor of prognosis in chronic obstructive pulmonary disease. Am J Respir Crit Care Med 2007; 175: 250-255.

38 de Torres JP, Pinto-Plata V, Casanova C, et al. C-reactive protein levels and survival in patients with moderate to very severe COPD. Chest 2008; 133: 1336-1343.

39 Mehrotra N, Freire AX, Bauer DC, et al. Predictors of mortality in elderly subjects with obstructive airway disease: the PILE score. Ann Epidemiol 2010; 20: 223-232.

40 Liu SF, Wang CC, Chin $\mathrm{CH}$, et al. High value of combined serum C-reactive protein and BODE score for mortality prediction in patients with stable COPD. Arch Bronconeumol 2011; 47: 427-432.

41 Zhang J, Rutten FH, Cramer MJ, et al. The importance of cardiovascular disease for mortality in patients with COPD: a prognostic cohort study. Fam Pract 2011; 28: 474-481.

42 Høiseth AD, Omland T, Hagve TA, et al. NT-proBNP independently predicts long term mortality after acute exacerbation of COPD - a prospective cohort study. Respir Res 2012; 13: 97.

43 Deng ZC, Zhao P, Cao C, et al. C-reactive protein as a prognostic marker in chronic obstructive pulmonary disease. Exp Ther Med 2014; 7: 443-446.

44 Moberg M, Vestbo J, Martinez G, et al. Prognostic value of C-reactive protein, leukocytes, and vitamin D in severe chronic obstructive pulmonary disease. Sci World J 2014; 2014: 140736.

45 Cano NJ, Pichard C, Court-Fortune I, et al. Survival of patients with chronic respiratory failure on long-term oxygen therapy and or non-invasive ventilation at home. Clin Nutr 2015; 34: 739-744.

46 Ford ES, Cunningham TJ, Mannino DM. Inflammatory markers and mortality among US adults with obstructive lung function. Respirology 2015; 20: 587-593.

47 Kleber ME, Siekmeier R, Delgado G, et al. C-reactive protein and lipoprotein-associated phospholipase A2 in smokers and nonsmokers of the Ludwigshafen Risk and Cardiovascular Health study. Adv Exp Med Biol 2015; 832: 15-23.

48 Blumenthal JA, Smith PJ, Durheim M, et al. Biobehavioral prognostic factors in chronic obstructive pulmonary disease: results from the INSPIRE-II trial. Psychosom Med 2016; 78: 153-162.

49 Loprinzi PD, Walker JF. Increased daily movement associates with reduced mortality among COPD patients having systemic inflammation. Int J Clin Pract 2016; 70: 286-291.

50 Saltürk C, Karakurt Z, Adiguzel N, et al. Does eosinophilic COPD exacerbation have a better patient outcome than non-eosinophilic in the intensive care unit? Int J Chron Obstruct Pulmon Dis 2015; 10: 1837-1846.

51 Murphy SA, Haja Mydin H, Fatah S, et al. Predicting end-of-life in patients with an exacerbation of COPD by routine clinical assessment. Respir Med 2010; 104: 1668-1674.

52 Zhao YF, Jiang YP, Zhou LF, et al. The value of assessment tests in patients with acute exacerbation of chronic obstructive pulmonary disease. Am J Med Sci 2014; 347: 393-399. 
53 Stolz D, Christ-Crain M, Morgenthaler NG, et al. Plasma pro-adrenomedullin but not plasma pro-endothelin predicts survival in exacerbations of COPD. Chest 2008; 134: 263-272.

54 Duman D, Aksoy E, Agca MC, et al. The utility of inflammatory markers to predict readmissions and mortality in COPD cases with or without eosinophilia. Int J Chron Obstruct Pulmon Dis 2015; 10: 2469-2478.

55 Tofan F, Rahimi-Rad MH, Rasmi Y, et al. High sensitive C-reactive protein for prediction of adverse outcome in acute exacerbation of chronic obstructive pulmonary disease. Pneumologia 2012; 61: 160-162.

56 Zhang XL, Chi YH, Wang LF, et al. Systemic inflammation in patients with chronic obstructive pulmonary disease undergoing percutaneous coronary intervention. Respirology 2014; 19: 723-729.

57 Andreassen SL, Liaaen ED, Stenfors N, et al. Impact of pneumonia on hospitalizations due to acute exacerbations of COPD. Clin Respir J 2014; 8: 93-99.

58 Guertler C, Wirz B, Christ-Crain M, et al. Inflammatory responses predict long-term mortality risk in community-acquired pneumonia. Eur Respir J 2011; 37: 1439-1446.

59 Moghbeli N, Kirtane AJ, Ray KK, et al. C-reactive protein and cardiovascular outcomes in smokers versus nonsmokers in non-ST-elevation acute coronary syndrome (from the TACTICS-TIMI 18 trial). Am J Cardiol 2005; 96: 635-638.

60 Haja Mydin H, Murphy S, Clague H, et al. Anemia and performance status as prognostic markers in acute hypercapnic respiratory failure due to chronic obstructive pulmonary disease. Int J Chron Obstruct Pulmon Dis 2013; 8: 151-157.

61 Emerging Risk Factors Collaboration. C-reactive protein concentration and risk of coronary heart disease, stroke, and mortality: an individual participant meta-analysis. Lancet 2010; 375: 132-140.

62 Cozlea DL, Farcas DM, Nagy A, et al. The impact of C reactive protein on global cardiovascular risk on patients with coronary artery disease. Curr Health Sci J 2013; 39: 225-231.

63 Shrotriya S, Walsh D, Bennani-Baiti N, et al. C-reactive protein is an important biomarker for prognosis tumor recurrence and treatment response in adult solid tumors: a systematic review. PLoS One 2015; 10; e0143080.

64 Garcia-Rio F, Miravitlles M, Soriano JB, et al. Systemic inflammation in chronic obstructive pulmonary disease: a population-based study. Respir Res 2010; 11: 63.

65 Pinto-Plata VM, Müllerova H, Toso JF, et al. C-reactive protein in patients with COPD, control smokers and non-smokers. Thorax 2006; 61: 23-28.

66 Donaldson GC. C-reactive protein: does it predict mortality? Am J Respir Crit Care Med 2007; 175: 209-210.

67 Lomholt FK, Laulund AS, Bjarnason NH, et al. Meta-analysis of routine blood tests as predictors of mortality in COPD. Eur Clin Respir J 2014; 1 [DOI: 10.3402/ecri.v1.24110].

68 Hogg JC, Chu F, Utokaparch S, et al. The nature of small-airway obstruction in chronic obstructive pulmonary disease. N Engl J Med 2004; 350: 2645-2653.

69 Sin DD, Man SF. Why are patients with chronic obstructive pulmonary disease at increased risk of cardiovascular diseases? The potential role of systemic inflammation in chronic obstructive pulmonary disease. Circulation 2003; 107: 1514-1519.

70 Suwa T, Hogg JC, Quinlan KB, et al. Particulate air pollution induces progression of atherosclerosis. J Am Coll Cardiol 2002; 39: 935-942.

71 Camilli AE, Robbins DR, Lebowitz MD. Death certificate reporting of confirmed airways obstructive disease. Am J Epidemiol 1991; 133: 795-800.

72 McGarvey LP, John M, Anderson JA, et al. Ascertainment of cause-specific mortality in COPD: operations of the TORCH Clinical Endpoint Committee. Thorax 2007; 62: 411-415.

73 Chang CL, Robinson SC, Mills GD, et al. Biochemical markers of cardiac dysfunction predict mortality in acute exacerbations of COPD. Thorax 2011; 66: 764-768.

74 Akiki Z, Fakih D, Jounblat R, et al. Surfactant protein D, a clinical biomarker for chronic obstructive pulmonary disease with excellent discriminant values. Exp Ther Med 2016; 11: 723-730.

75 Mannino DM, Tal-Singer R, Lomas DA, et al. Plasma fibrinogen as a biomarker for mortality and hospitalized exacerbations in people with COPD. Chronic Obstr Pulm Dis 2015; 2: 23-34.

76 Stolz D, Meyer A, Rakic J, et al. Mortality risk prediction in COPD by a prognostic biomarker panel. Eur Respir J 2014; 44: 1557-1570.

77 Boeck L, Soriano JB, Brusse-Keizer M, et al. Prognostic assessment in COPD without lung function: the B-AE-D indices. Eur Respir J 2016; 47: 1635-1644.

78 Subramanian, Ragulan, Jindal A, et al. The study of efficacy, tolerability and safety of theophylline given along with formoterol plus budesonide in COPD. J Clin Diagn Res 2015; 9: OC10-OC13.

79 Ridker PM, Cannon CP, Morrow D, et al. C-reactive protein levels and outcomes after statin therapy. $N$ Engl J Med 2005; 352: 20-28.

80 Lee TM, Lin MS, Chang NC. Usefulness of C-reactive protein and interleukin-6 as predictors of outcomes in patients with chronic obstructive pulmonary disease receiving pravastatin. Am J Cardiol 2008; 101: 530e5.

81 Young RP, Hopkins R, Eaton TE. Pharmacological actions of statins: potential utility in COPD. Eur Respir Rev 2009; 118: 222-232.

82 Pavasini R, Biscaglia S, d'Ascenzo F, et al. Antiplatelet treatment reduces all-cause mortality in COPD patients: a systematic review and meta-analysis. COPD 2016; 13: 509-514.

83 Snow V, Lascher S, Mottur-Pilson C, et al. Evidence base for management of acute exacerbations of chronic obstructive pulmonary disease. Ann Intern Med 2001; 134: 595-599.

84 Dev D, Wallace E, Sankaran R, et al. Value of C-reactive protein measurements in exacerbations of chronic obstructive pulmonary disease. Respir Med 1998; 92: 664-667.

85 Ruiz-Gonzalez A, Lacasta D, Ibarz M, et al. C-reactive protein and other predictors of poor outcome in patients hospitalized with exacerbations of chronic obstructive pulmonary disease. Respirology 2008; 13: 1028-1033. 